riding will benefit the patient materially, considerable harm might be done if the patient had to be subjected to the conscious mental strain of re-learning to maintain his balance.

In my experience of treating the recoverable injured it is neither necessary nor advisable at any stage of treatment to focus attention upon digital dexterity, unless this be done to distract attention from the exercise of muscles other than those concerned in the particular act.

From the inception of treatment occupational therapy must be used to keep the uninjured muscles of the body up to their normal standard of development, and also to help the muscles in the injured part in their process of healing and regeneration. The work done must stop short of actual fatigue, but it is almost more important that it shall not allow periods of idleness. This will mean that some part of the time only uninjured muscles of the body will be exercised deliberately, but that the injured part of the body will never be exercised alone.

To obtain its maximum success occupational therapy should be continued throughout the day. It cannot be too often repeated that "getting fit is a whole-time job" and, in the words of William Cowper, "absence of occupation is not rest."

\title{
TREATMENT OF MINOR INJURIES OF THE ANKLE
}

\author{
By NORMAN ROBERTS, M.CH. ORTh., F.R.C.S.(Eng.) \\ Hon. Asst. Orth. Surg. Liverpool Royal Infirmary, Asst. Orth. Surg. North Wales \\ Sanatorium, etc.
}

\section{Stability of the Astragalus after Injury}

In recent years attention has been drawn to the frequency with which severe sprains of the external ligament of the ankle are complicated by a tilting deformity of the astragalus which can be demonstrated by X-rays taken with the foot fully inverted at the heel. If the condition is recognised at the time of the first injury there is a good prospect that by adequate fixation in plaster to relax the torn ligament, the sequel of repeated subluxations of the astragalus may be avoided. The lesson to be learnt from this type of injury can be applied to the treatment of all ankle injuries, whether sprains or fractures.

The layman and sometimes the doctor has said in the past that "a sprain of the ankle is worse than a fracture." If by fracture is meant the commonest type of injury, an uncomplicated fracture of the external malleolus without displacement, then the statement is certainly true. The greater pain and swelling of a severe sprain and the prospect of future trouble are due to the fact that the completely ruptured external lateral ligament has prejudiced the stability of the astragalus. In contrast, bad results and a slow recovery from simple fractures of the external malleolus without displacement are much more likely to follow over-zealous treatment than neglect. Modern teaching in fracture treatment has been so insistent on the necessity for "absolute and complete immobilisation until the fracture has united" that any person who sustains a simple crack fracture nowadays is lucky to escape imprisonment of the part in plaster of Paris for six to eight weeks. The fault does not lie so much with the teaching as with its misinterpretation.

In simple language, there are only two indications for fixation or immobilisation of an injured part. The first is to keep the broken bone or sprained ligament in position, and the other is to make it unite. It is well known that many fractures will unite whatever is done to them. It should also be well known that many simple fractures without displacement are self-splinting and, short of further injury or violence, cannot displace. It follows that if in any given fracture or sprain there is no danger of non-union or of displacement, immobilisation in the true sense of the word is both unnecessary and harmful. To take an example, one does not "immobilise" an impacted fracture of the neck of the humerus." In fact, one makes every effort to start shoulder movements as early as possible in order to minimise stiffness in the shoulder joint. In short, where a fracture or other injury is not in real need of immobilisation, the stiffness and disuse change which result from unnecessary plaster fixation can be avoided by using simpler methods of treatment. Bohler's teaching in relation to the walking plaster is that in the lower limb functional activity while the plaster is on prevents stiff joints and wasted muscles. Would that this were true. Since it is not, we must still try to spare our patients the harmful effects of plaster fixation in those cases where one can get away from the necessity for immobilisation. It is hoped in this article, using the example of injuries of the ankle, to show how far this is possible. 


\section{Sprains of the Ankle}

Internal Lateral Ligament.-The internal lateral ligament is strong and thick. It may rupture when the astragalus displaces outwards in a Pott's fracture, but sprains of the ligament without fracture are uncommon. If an abduction levering strain falls on the inner side of the ankle it is more likely to cause an isolated fracture of the internal malleolus than to tear the internal lateral ligament. If the internal lateral ligament is sprained there will be swelling and tenderness below the tip of the malleolus and pain on everting the foot. An X-ray should be taken with the heel fully everted, and strong tension on the inner side of the ankle joint. If this does not reveal any tilting or instability of the astragalus the support of strapping in the inverted position for a few weeks is all that is necessary.

If the X-ray reveals any outward tilting of the astragalus, and especially if there is also spreading at the tibio-fibular joint indicating rupture of the tibio-fibular ligaments, plaster fixation is necessary. The foot should be moderately inverted at the heel and a walking plaster applied and kept on for a period of about six weeks. After the plaster is removed the heel of the shoe should be crooked and elongated on the inner side as a temporary measure.

External Lateral Ligament.-Sprains of this ligament are very common. They are caused by a violent inversion movement of the foot, and the anterior and middle bands of the ligament are strained or ruptured. It is most important that an antero-posterior X-ray should be taken with the foot held strongly inverted at the heel. In cases of complete rupture of the

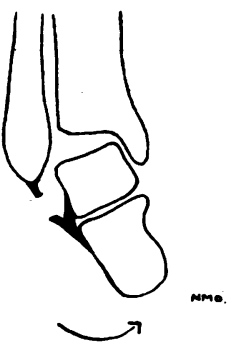

FIG. I.-X-ray in full inversion showing tilting of the astragalus due to complete rupture of the external lateral ligament.

ligament tilting of the astragalus up to an angle of thirty degrees from the horizontal may show in the X-ray. If any doubt is felt about the lesser degrees of tilting a comparison X-ray of the other ankle should be taken in the inverted position. A slight amount of tilting may be normal to the particular individual concerned, and the comparison $\mathrm{X}$-ray is therefore valuable

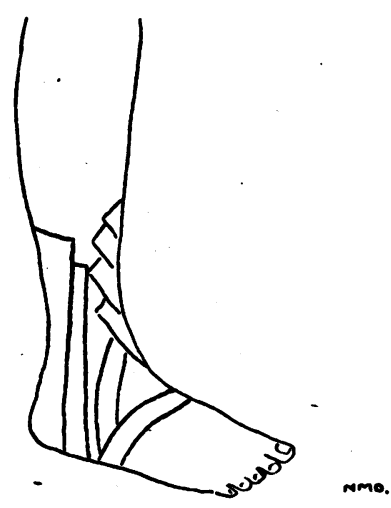

(a)

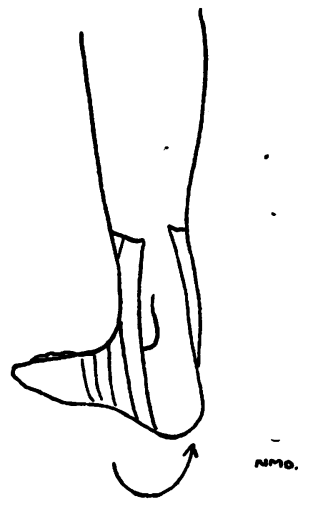

(b)

Fig. 2.-Method of strapping for minor sprains and fractures without instability of the astragalus. 
in border-line cases. If any difficulty is experienced in inverting the foot strongly because of pain a local anaesthetic should be injected below the tip of the malleolus.

If tilting is demonstrated in the $\mathrm{X}$-ray and there is no previous history of sprains of the ankle a plaster cast must be applied with the foot in the moderately everted position, so as to relax the torn ligament. The period of fixation will vary from six to ten weeks according to circumstances. If the ankle has been sprained before it is likely that the instability is due to the old injury and not to the recent one, therefore plaster fixation will do nothing to repair the torn ligament and strapping support is all that is necessary.

If the sprain is a simple one, and there is no tilting of the astragalus, the foot is strapped up in the everted position. Ordinary zinc oxide strapping in strips two inches broad is preferable to brown holland strapping or elastoplast. The strapping can be applied very neatly in the form of a U-shaped stirrup, leaving the tendo Achillis free so as to allow a little up and down movement of the ankle, while at the same time preventing lateral strains.

Chronic Sprains of the External Lateral Ligament.-The commonest cause of recurrent sprains of the ankle is a torn external lateral ligament which has never repaired properly. In a young person in whom there háve been repeated inversion sprains and where tilting of the astragalus can be demonstrated in an X-ray, operative treatment is ideal. The operation to form a new external lateral ligament using the peroneus brevis tendon, as described by Watson-Jones, is very effective in curing these cases. If there is any contra-indication to operation, or if recurrent sprains are not accompanied by any sign of instability of the astragalus the outside of the heel of the shoe should be crooked and elongated, and the patient should wear a supporting elastic anklet.

\section{A. Minor Fractures.}

\section{Fractures of the Ankle}

I. External Rotation Fractures.-The commonest fracture of the ankle is a first degree external rotation spiral fracture of the external malleolus. Nearly half of the total cases of fracture fall into this group. The clinical signs are similar to those of a sprain on the outer side of the ankle, and it is often difficult to decide without an X-ray whether there is a fracture or not. In the majority of the cases there is no sign of any damage on the inner side of the ankle, which is completely free from any tenderness or swelling. Before deciding about the method of treatment to be employed one must test for stability of the astragalus in exactly the same way as has been described in the treatment of sprains. The clinical test of

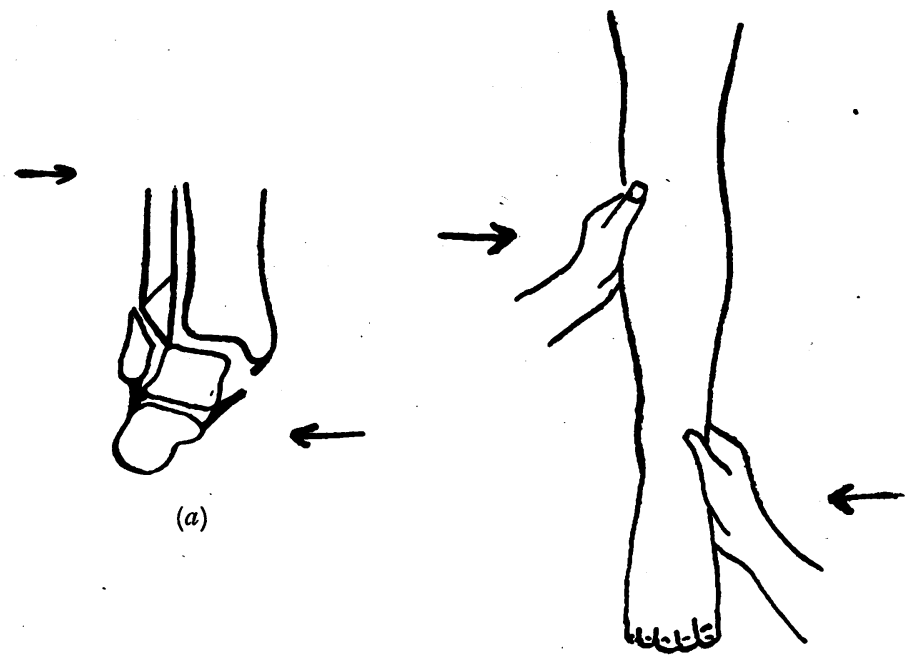

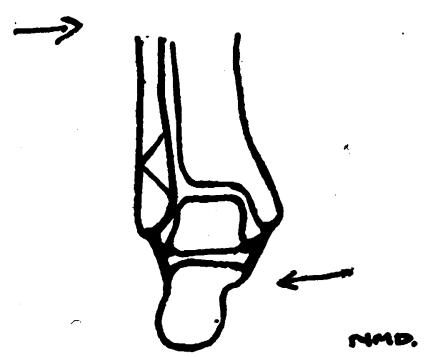

(c)

(b)

FIG. 3.-Method of testing for stability in external rotation fractures of the external malleolus. The small figure on the left illustrates an unstable astragalus with rupture of the internal lateral ligament. The small figure on the right shows a stable astragalus without damage on the inner side of the ankle. 
stability is reliable if properly performed, but it can be supplemented if necessary by an X-ray. The inner side of the heel is grasped firmly with one hand, and the leg is steadied just below the knee with the opposite hand on the lateral side of the limb. Firm and increasing pressure is now applied to the astragalus in a deliberate attempt to push it laterally. If there is no damage to the internal lateral ligament or internal malleolus this manoeuvre causes no pain in a simple fracture of the external malleolus and at once indicates that the fracture is self-splinting. This is of great importance, since the $\mathrm{X}$-ray appearance alone may be misleading. The greatest danger lies in those cases where there has been complete rupture of the internal lateral ligament. By the time the case is seen spontaneous reduction has occurred, and the X-ray looks innocent with a simple fracture of the external malleolus and no displacement. Clinical examination will at once reveal this type of injury as a major and important injury. There will be tenderness and swelling on the inner side of the ankle, and any attempt to push the astragalus laterally, even gently, will cause pain, and may even cause displacement if the pressure is applied incautiously.

In the first group of fractures where there is no injury on the inner side of the ankle there is no need for plaster fixation. The patient is more comfortable in strapping, and will be able to walk within a few days. Complete recovery occurs within four or five weeks, and disuse change is very much less than if plaster fixation is employed unnecessarily. The second group of injuries falls into the same class as a complete Pott's fracture with displacement, and should receive a generous period of fixation in plaster for a minimum of eight weeks, and if necessary as long as twelve weeks in heavily built patients. If fixation is not employed for long enough, and the patient walks on an unsoundly united fracture, the astragalus will start to drift into the valgus position.

2. Abduction Fractures.--In this type of injury there is an isolated transverse fracture of the internal malleolus as the result of an abduction strain applied to the inner side of the ankle. If the injury is more severe the next point of damage is rupture of the tibiofibular ligaments with diastasis, and finally a fracture of the shaft of the fibula three or four inches above the ankle. In severe cases with marked outward displacement of the astragalus the latter bone rides up between the tibia and fibula producing the so-called Dupuytren's fracture. We are concerned here only with the first stage of the injury, an isolated fracture of the internal malleolus. This injury corresponds roughly in severity to the fracture of the external malleolus described above. Pain, tenderness and swelling are strictly localised to the inner side of the ankle, and the astragalus is in most cases quite stable and shows no tendency to tilting into valgus. One meets with many of these cases which have been treated as sprains without the knowledge of there being any fracture, and despite the lack of support and fixation displacement does not occur. Unless there is any very marked degree of swelling or demonstrable instability of the astragalus when the heel is pushed into eversion firm support by strapping is quite sufficient, and plaster fixation for many weeks is both unnecessary and harmful. The danger of treating these cases in strapping rather than plaster lies in the case which has a rupture of the tibio-fibular ligaments, and therefore a potentially unstable astragalus. As in the case of fracture of the outer side of the ankle, the tendency to instability can be recognised both by clinical examination and by X-rays and plaster fixation employed where it is necessary.

3. Inversion Sprain Fractures of the External Malleolus.-This type of injury is produced by a forcible inversion sprain. - The external malleolus may be fractured transversely

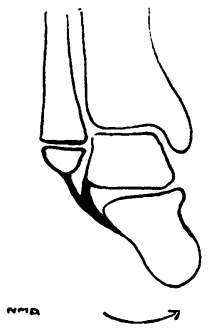

FIG. 4.-Inversion fracture of the external malleolus. $X$-rays in inversion show no tilting or instability Plaster fixation is unnecessary. 
at any point from its tip up to the level of the ankle joint. Most of the cases show no displacement, and should be regarded and treated in exactly the same way as sprains of the external lateral ligament. If there is instability and tilting of the astragalus when the foot is $\mathrm{X}$-rayed in inversion then immobilisation is necessary. If there is not, strapping support will be sufficient.

4. Novocain Injections in the Treatment of Sprains and Fractures.-It has been recommended that in minor injuries to the ankle and elsewhere that novocain solution should be injected at the point of swelling and tenderness. It is claimed that this method allows a quicker and more painless return to functional activity, and that the amount of swelling and stiffness is diminished by the injection. The method is an attractive one. It has two chief drawbacks, the first being the rather remote danger of sepsis, and the second the danger of masking the true nature of the injury by making it painless. The first objection should not apply if the usual aseptic precautions are carefully observed. The second objection is a serious one. In the hands of the experienced surgeon, who is able to discriminate between the potentially dangerous injuries and the simpler ones, the method has much to commend it. Applied indiscriminately it can be a double-edged weapon. The quick recoveries claimed for the injection of novocain are much more likely to be due to the absence of fixation and the early movements employed than to any direct effect of the injection, and where novocain is not employed a similarly quick recovery can be observed provided plaster of Paris is not used unnecessarily in the minor injuries. It seems to the author that the chief use of the method lies in its capacity to make the injured point painless so that one can have a free hand to test clinically and by X-rays for any potential instability.

\title{
B. Major Fractures.
}

The chief purpose of this article has been to draw attention to those minor injuries of the ankle, including fracture, which can safely and profitably be treated without immobilisation in plaster. The treatment of the more serious degrees of fracture with dislocation and displacement is not within the scope of this article. Suffice it to say that the tendency is to overtreat the minor injuries and to undertreat the major injuries. The "six to eight weeks in plaster" prescription is often applied with equal zeal to crack fractures in the malleolus as it is to a thoroughgoing Pott's fracture with marked displacement. It is hoped that a proper appreciation of the minor injuries will lead to a greater respect for the major injuries.

\section{TUBERCULOSIS OF THE KNEE JOINT}

\author{
By R. Y. PATON, M.A., F.R.C.S.(ENG.) \\ (Surg. Royal National Orthopaedic Hospital, etc.)
}

The knee-joint is a common site of tuberculous infection, second only to the hip-joint in frequency. The infection may arise at any age, but usually starts in childhood, and sometimes even in the first months of life. The slow, insidious development of a swelling of a knee-joint, with limitation of movement and pain on attempting movement must always be regarded with suspicion, and the knee treated as tuberculous until the diagnosis is finally settled. There may be a history of a strain or twist of the joint preceding the development of the symptoms, but it must always be borne in mind that such trauma may have been the means of producing these symptoms in a joint with a pre-existing tuberculous infection. On closer investigation, however, it is usually found that the trauma has nothing to do with the onset of the disease. The knee, as a result of its anatomical construction, whereby two large bones are but poorly adapted the one to the other, is always the subject of strain in normal activity and exercise, and the fact that the knee suddenly becomes painful during such exercise obviously does not mean that the injury has caused the tuberculous infection to settle down suddenly in the joint. The strain has brought to the notice of the patient the presence of an infection. Much more frequently the onset is very slow and gradual, and pain may be markedly delayed in making 